\title{
Phase II Study of Weekly Amrubicin for Refractory or Relapsed Small Cell Lung Cancer
}

\author{
HIROSHIGE YOSHIOKA ${ }^{1,2,3, *}$, YOSHIHITO KOGURE ${ }^{4,5, *}$, MASAHIKO ANDO $^{6}$, \\ CHIYOE KITAGAWA ${ }^{4}$, MASAHIRO IWASAKU ${ }^{1,7}$, TAKASHI NIWA ${ }^{1,8}$ and HIDEO SAKA ${ }^{4}$ \\ ${ }^{1}$ Department of Respiratory Medicine, Kurashiki Central Hospital, Kurashiki, Japan; \\ ${ }^{2}$ Clinical Research Center, and Departments of ${ }^{4}$ Respiratory Medicine and \\ ${ }^{5}$ Medical Oncology, National Hospital Organization Nagoya Medical Center, Nagoya, Japan; \\ ${ }^{3}$ Department of Thoracic Oncology, Kansai Medical University Hospital, Hirakata, Japan; \\ ${ }^{6}$ Center for Advanced Medicine and Clinical Research, Nagoya University Hospital, Nagoya, Japan; \\ ${ }^{7}$ Department of Pharmacoepidemiology, Graduate School of Medicine and Public Health, Kyoto University, Kyoto, Japan; \\ ${ }^{8}$ Department of Respiratory Medicine, Kanagawa Cardiovascular and Respiratory Center, Yokohama, Japan
}

\begin{abstract}
Background: Amrubicin hydrochloride is administered as second- or third-line therapy for small cell lung cancer, and is known to cause severe myelotoxicity. This study evaluated the efficacy and safety of weekly amrubicin for refractory/relapsed small cell lung cancer. Patients and Methods: A single-arm, open-label, multicenter, phase II study of weekly amrubicin was performed in 21 patients at seven centers in Japan from 2012 through 2015. Results: A partial response (PR) was noted in one out of the first 18 patients. The study was terminated early according to the termination criteria in the protocol. In total, the response rate was 19\% (no complete responses and four PRs) and the disease control rate was $81 \%$ (17/21). Median overall survival was 288 days (95\% confidence interval $(C I)=208$ 424 days), while median progression-free survival was 113 days (95\% CI=45-202 days). Conclusion: This study failed to demonstrate any efficacy of weekly amrubicin for refractory/relapsed small cell lung cancer.
\end{abstract}

Small cell lung cancer accounts for about $15 \%$ of all lung cancers and is known to have a very poor prognosis. Despite

This article is freely accessible online.

*These Authors contributed equally to this study.

Correspondence to: Hiroshige Yoshioka, MD, Ph.D., Department of Thoracic Oncology, Kansai Medical University Hospital, Hirakata, Japan, 3-1 Shinmachi 2 Chome, Hirakata City, Osaka 573-1191, Japan. Tel.: +81 728040101, Fax: +81 728042504, e-mail: hgyoshioka@gmail.com

Key Words: Amrubicin, small cell lung cancer, refractory, relapsed, phase II. advances in chemotherapy, patients with advanced small cell lung cancer still have a median survival time of 9 to 12 months and a 2-year survival rate of about 5-20\%. Accordingly, development of more effective chemotherapy is required.

Amrubicin hydrochloride was developed in Japan and is a derivative of the anthracycline doxorubicin hydrochloride. In Japan, Europe, and the United States, it has been reported that amrubicin hydrochloride has a good antitumor activity against small cell lung cancer (1-5). It is frequently administered alone as second-line or third-line therapy for small cell lung cancer, but is known to cause severe myelotoxicity.

In clinical practice, amrubicin hydrochloride is usually administered once daily for 3 consecutive days, every 3 weeks (1). For other medications, it has been suggested that toxicity can be reduced to a low level without attenuating efficacy by dividing the dose and performing weekly administration (68). A weekly dosing regimen of amrubicin hydrochloride might reduce side-effects including myelosuppression, which is severe with the conventional regimen. As a result, there is a possibility that the dose intensity and effectiveness of treatment could be improved. It is also possible that the weekly regimen may be more convenient for patients by reducing the number of hospital visits. Therefore, as a new administration regimen for amrubicin hydrochloride in patients with previously treated refractory or relapsed lung cancer, weekly administration was assessed in a dose-finding phase I study. As a result, it was concluded that the maximum tolerated dose was $65 \mathrm{mg} / \mathrm{m}^{2}$ and the recommended dose was $60 \mathrm{mg} / \mathrm{m}^{2}$ (9). In order to further investigate the efficacy and safety of weekly amrubicin hydrochloride as second-line therapy for refractory or relapsed small cell lung cancer, we planned a phase II study using the recommended dose from the phase I study. 
Table I. Eligibility criteria for study patients.

Inclusion criteria

1. Patients with small cell lung cancer confirmed by cytodiagnosis or histology.

2. Patients indicated for systemic chemotherapy and refractory/relapsed after primary platinum-containing therapy (primary treatment included postoperative chemotherapy if it was platinum-containing therapy).

3. Patients who had not had prior treatment with amrubicin hydrochloride.

4. Patients aged 20 years or older (without upper limit).

5. Patients with an Eastern Cooperative Oncology Group Performance Status of 0 to 2.

6. Patients with measurable lesions (RECIST 1.1).

7. Patients with adequate function of major organs:

- Leukocyte count $\geq 3,000 / \mu l$

- Neutrophil count $\geq 1,500 / \mu l$

- Hemoglobin $\geq 9 \mathrm{~g} / \mathrm{dl}$

- Platelet count $\geq 100,000 / \mu 1$

$-\mathrm{AST} \leq 100 \mathrm{IU} / 1$

$-\mathrm{ALT} \leq 100 \mathrm{IU} / 1$

- Total bilirubin $\leq 1.5 \mathrm{mg} / \mathrm{dl}$

- Serum creatinine $\leq 1.5 \mathrm{mg} / \mathrm{dl}$

- $\mathrm{SpO} 2 \geq 92 \%$ (while breathing ambient air)

8. Patients with asymptomatic brain metastasis were eligible, excluding those who required steroid therapy for brain metastasis during the study period.

9. Patients with recurrence after surgery for the primary tumor or more than 42 days after radical irradiation of the primary tumor.

10. Patients who received palliative radiotherapy or surgery for local recurrence/metastasis more than 14 days previously.

11. Patients who finished prior chemotherapy more than 28 days previously.

12. Patients expected to survive for more than 3 months from the scheduled start of administration.

13. Patients who were able to provide written consent.

Exclusion criteria

1. Patients for whom amrubicin hydrochloride was contraindicated.

2. Patients with carcinomatous pericarditis, pleurisy, or peritonitis for which local therapy was indicated. However, patients were registered if their condition was controlled and it was more than 2 weeks after local therapy. (If a drain tube was inserted, the patient could be registered more than 2 weeks after removal of the drain. Picibanil, minomycin, and talc were acceptable as sclerosants, but cisplatin and other anticancer agents were not allowed.)

3. Patients receiving radiation therapy. (Those who satisfied eligibility criteria 9 or 10 were registered after completion of radiotherapy.)

4. Patients with symptomatic brain metastasis. (See eligibility criterion 8.)

5. Patients with superior vena cava syndrome. (Registration possible if eligibility criterion 10 was met after palliative radiotherapy.)

6. Patients with cardiac dysfunction (confirmed electrocardiographic abnormalities requiring treatment, myocardial infarction within 6 months before enrollment, or poorly controlled angina or heart failure).

7. Patients with interstitial pneumonia or pulmonary fibrosis confirmed on chest X-ray. (Patients with radiation pneumonitis limited to the site of irradiation could be registered.)

8. Patients with a complication of poorly controlled diabetes.

9. Patients whose prior treatment with cardiotoxic agents, including other anthracyclines, reached the limits shown below:

- Daunorubicin hydrochloride: Total dose of $25 \mathrm{mg} / \mathrm{kg}$ body weight

- Doxorubicin hydrochloride: Total dose of $500 \mathrm{mg} / \mathrm{m}^{2}$ body surface area

- Epirubicin hydrochloride: Total dose of $900 \mathrm{mg} / \mathrm{m}^{2}$ body surface area

- Pirarubicin hydrochloride: Total dose of $950 \mathrm{mg} / \mathrm{m}^{2}$ body surface area

10. Patients with active double cancer, excluding carcinoma in situ and other cancer without recurrence for more than 5 years.

11. Patients with infection or suspected infection and fever (temperature $>38^{\circ} \mathrm{C}$ ).

12. Women who were pregnant, possibly pregnant, breastfeeding, or refused to use effective contraception.

13. Other patients for whom it was judged that participation in this study would be difficult by the principal investigator or investigators due to serious complications.

ALT, Alanine aminotransferase; AST, aspartate aminotransferase.

In this phase II study, the efficacy and safety of weekly amrubicin therapy was assessed for small cell lung cancer that was refractory to first-line chemotherapy or had relapsed.

\section{Patients and Methods}

Patients. Patients who satisfied the eligibility criteria (Table I) were enrolled. Refractory relapse was defined as no response to first-line 


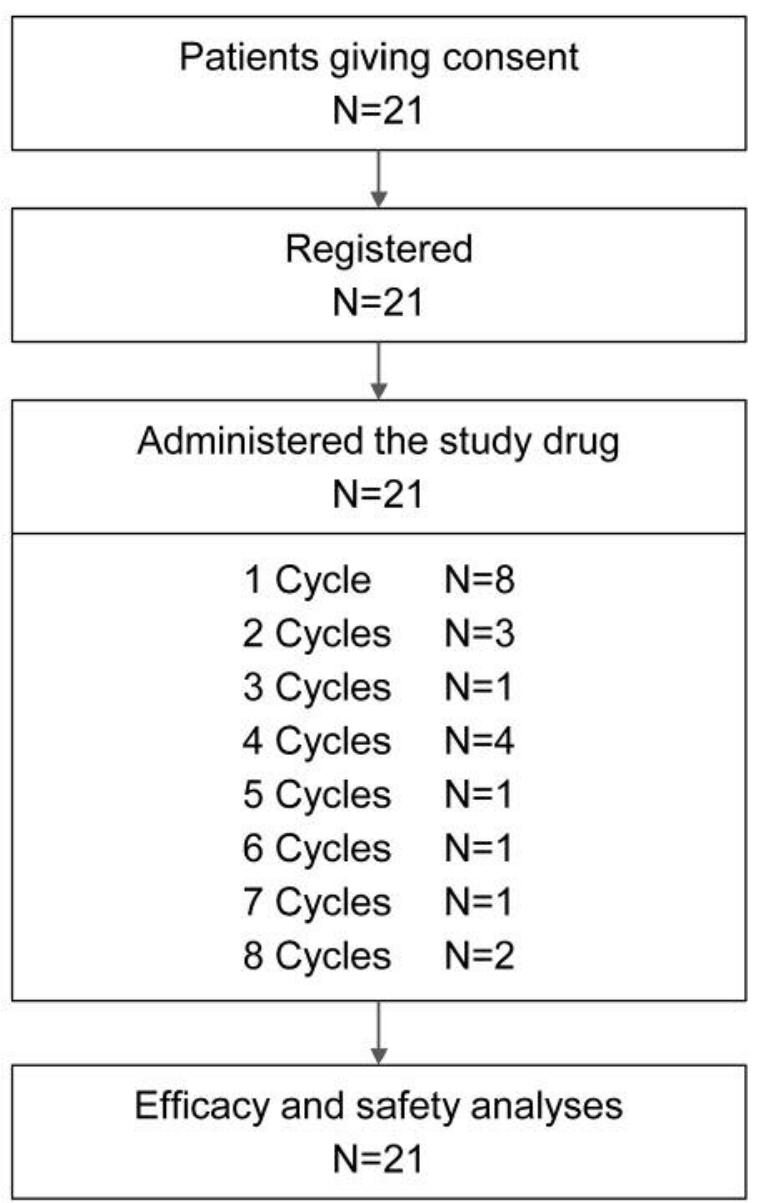

Figure 1. Participant flow chart.

chemotherapy or relapse within 90 days after the date of the final dose of first-line chemotherapy, while sensitive relapse was defined as relapse 90 days or more after the date of the final dose of firstline chemotherapy.

Trial design and treatment. This was a single-arm, open-label, multicenter, phase II study. It was registered with the UMIN Clinical Trials Registry (UMIN-CTR, URL: http://www.umin.ac.jp/ ctr/): registration number UMIN000009440. The study protocol was reviewed and approved by each Institutional Review Board, and written informed consent was obtained from all of the patients. Amrubicin was administered at a dose of $60 \mathrm{mg} / \mathrm{m}^{2}$ on days 1 and 8 , and this regimen was repeated every 3 weeks until disease progression or development of intolerable toxicity.

Endpoints. The primary endpoint was the response rate as defined by the Response Evaluation Criteria in Solid Tumors (RECIST), version 1.1 (10). Confirmed partial response (PR) was defined as PR also on the second computed tomographic evaluation at 4 weeks or more after the initial detection of PR. Secondary endpoints were progression-free (PFS) and overall (OS) survival, and the incidence of adverse events. PFS was defined as the time from the day of
Table II. Demographic characteristics of study participants.

\begin{tabular}{lc}
\hline Characteristic & $\mathrm{n}(\%)$ \\
\hline Total & $21(100)$ \\
Gender & $18(85.7)$ \\
Male & $3(14.3)$ \\
Female & \\
Age (years) & 67 \\
Median & $49-81$ \\
Range & \\
Stage at diagnosis & $3(14.3)$ \\
IIIa & $3(14.3)$ \\
IIIb & $15(71.4)$ \\
IV & $16(76.1)$ \\
Disease extent & $5(23.8)$ \\
Extensive & $21(100)$ \\
Limited & $0(0)$ \\
Smoking history & \\
Yes & $17(81)$ \\
No & $4(19)$ \\
Relapse type & \\
Sensitive & Refractory
\end{tabular}

enrollment to the day that disease progression was confirmed by imaging studies or the day of death from any cause, whichever came first. OS was defined as the time from the day of enrollment to the day of death from any cause, and patients were censored on the last date of survival was confirmed. Adverse events were monitored and graded according to the National Cancer Institute Common Terminology Criteria for Adverse Events (CTCAE), version 4.0 (11).

Statistical analysis. By assuming that efficacy would be equal to or higher than that achieved with conventional administration $(2,3)$, and assuming that the threshold response rate was $20 \%$, the expected response rate was $40 \%, \alpha=0.05$ (one-sided), and $\beta=0.2$, it was calculated that 18 patients were required for the first stage of the Simon two-stage theory (Minimax model). If there were at least five responders among the initial 18 patients, 15 more patients would be added at the second stage. If 11 responders were obtained among the total of 33 patients, this regimen would be judged to be effective. In consideration of possible inappropriate enrollments, two more patients were added, and the target number of patients was therefore set as 35 .

According to the protocol, this therapy would be defined as ineffective if a response of PR or better was achieved by fewer than five of the initial 18 patients, resulting in termination of the study. The response rate, PFS, OS, and safety (incidence of adverse events) were analyzed.

\section{Results}

Twenty-one patients from seven centers in Japan were enrolled during the period from December, 2012 through May, 2015. The participant flow chart is shown in Figure 1.

Baseline data are listed in Table II. Twenty-one patients were registered, among whom 18 were men. Their median 
Table III. Efficacy of amrubicin hydrochloride in study patients.

\begin{tabular}{lc}
\hline & $\mathrm{n}(\%)$ \\
\hline $\mathrm{CR}$ & $0(0)$ \\
$\mathrm{PR}$ & $4(19)$ \\
$\mathrm{SD}$ & $13(62)$ \\
$\mathrm{PD}$ & $3(14)$ \\
$\mathrm{NE}$ & $1(5)$ \\
Total & $21(100)$ \\
\hline
\end{tabular}

CR, Complete response; PR, partial response; SD, Stable disease; PD, progressive disease; NE, not evaluable.

age was 67 years (range=49-81 years). All patients had a history of smoking. At initial diagnosis, only five patients had limited disease. The tumor showed refractory recurrence in four patients and sensitive recurrence in 17.

PR was achieved in one out of first 18 patients. As recommended by the independent Data and Safety Monitoring Committee, the trial was terminated because it did not satisfy the criteria (PR in five or more of the first 18 patients) for proceeding to the second step according to the Simon two-stage theory.

The number of cycles administered ranged from 1 to 8 and the median number was 2 . The response rate was $19 \%$ (Table III). There was no patient with a complete response (CR) and four patients with $\mathrm{PR}$. The disease control rate $(\mathrm{PR}+\mathrm{CR}+$ $\mathrm{SD} /$ intention to treat population) was $81 \%(17 / 21)$. The median survival time was 288 days (Figure 2, 95\% confidence interval=208-424 days), and median PFS was 113 days (Figure 3, 95\% $\mathrm{CI}=45-202$ days).

A grade 3-4 decrease of the white blood cell count and absolute neutrophil count occurred in $11(55 \%)$ and 15 patients $(75 \%)$, respectively, while febrile neutropenia was found in four patients (20\%). Grade 3-4 anemia or thrombocytopenia was noted in eight patients (40\%) and three patients (15\%), respectively. Grade 3-4 elevation of aspartate transaminase, alanine transaminase, or creatinine occurred in one patient each (5\%), and grade 3-4 hyponatremia was observed in 3 patients (15\%). As non-hematological toxicities, grade 3-4 dyspnea and oral mucositis were found in one (5\%) and two patients (10\%), respectively. In addition, there were two cases (10\%) of grade 2 pneumonitis and 1 case $(5 \%)$ of grade 3 pneumonitis. There were no treatment-related deaths (Table IV).

\section{Discussion}

This study was terminated early due to the low response rate and further assessment of weekly administration of amrubicin was stopped. Inoue et al. reported a response rate of $67 \%$ for amrubicin in patients with sensitive relapse (12). Although

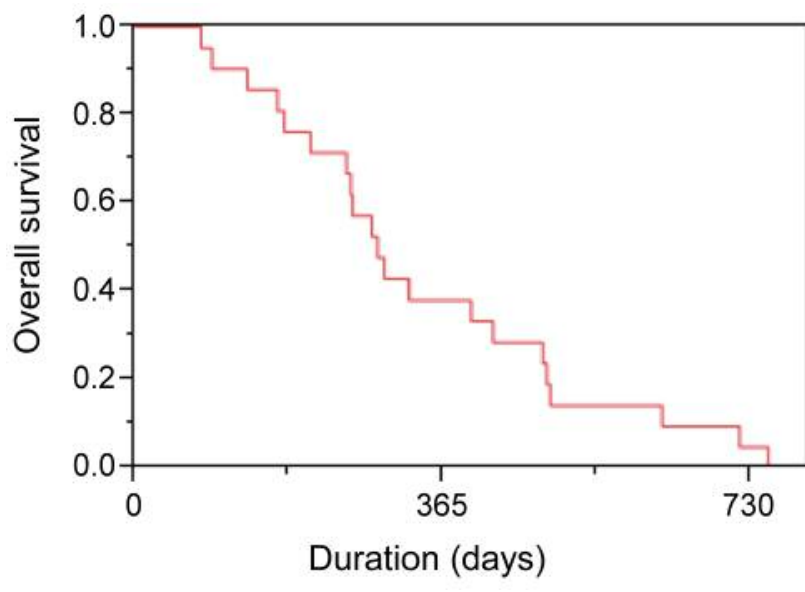

Figure 2. Overall survival of study patients on amrubicin hydrochloride.

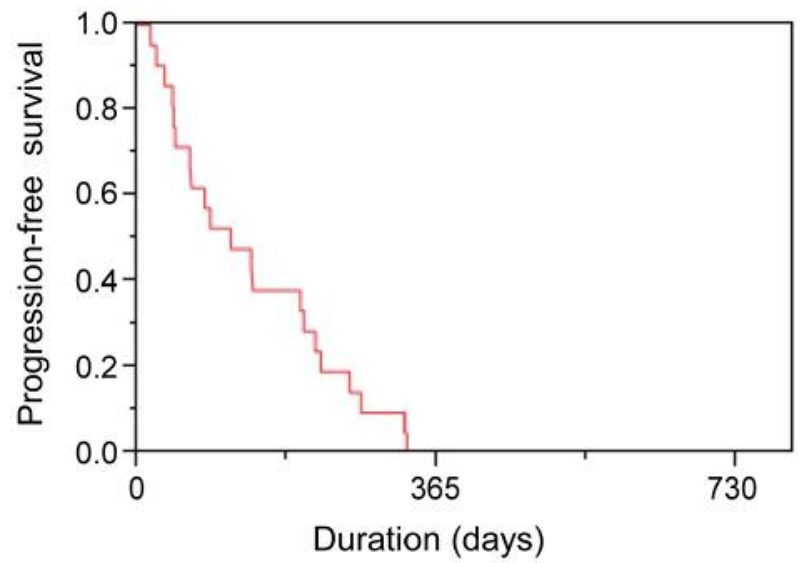

Figure 3. Progression-free survival of study patients on amrubicin hydrochloride.

$81 \%$ of the patients in our trial had sensitive relapse, the response rate was only $19 \%$ and we were unable to obtain a response rate as high as that of Inoue et al. Although the response rate of $19 \%$ was below expectations, weekly amrubicin achieved a high disease control rate, since $81 \%$ of the patients had stable disease or PR. Therefore, our results indicate that weekly administration of amrubicin hydrochloride was able to stabilize the disease, but was not as effective as the conventional regimen for obtaining responses.

Discontinuation of this trial might have been avoided if the disease control rate had been selected as the primary endpoint instead of the response rate. A possible reason for the low response rate in this study is that etoposide, which is a topoisomerase II inhibitor like amrubicin hydrochloride and which can induce cross-resistance, might have been used in prior treatment for many patients, as reported previously (13). 
Table IV. Adverse events experienced by study patients on amrubicin hydrochloride.

\begin{tabular}{|c|c|c|}
\hline & \multicolumn{2}{|c|}{ Total $n=21$} \\
\hline & All grade & Grade 3,4 \\
\hline \multicolumn{3}{|l|}{ Hematological } \\
\hline WBC & 17 & 11 \\
\hline Neutrophils & 18 & 15 \\
\hline $\mathrm{Hb}$ & 18 & 8 \\
\hline Plt & 14 & 3 \\
\hline Alb & 12 & 0 \\
\hline AST & 4 & 1 \\
\hline ALT & 4 & 1 \\
\hline Cre & 8 & 1 \\
\hline T.Bil & 5 & 0 \\
\hline Hyponatremia & 6 & 3 \\
\hline Hyperkalemia & 1 & 0 \\
\hline Hypokalemia & 0 & 0 \\
\hline Hypercalcemia & 1 & 0 \\
\hline Hypocalcemia & 9 & 0 \\
\hline \multicolumn{3}{|l|}{ Non-hematological } \\
\hline Pneumonitis & 6 & 1 \\
\hline Ventricular arrhythmia & 1 & 0 \\
\hline Nausea & 5 & 0 \\
\hline Vomiting & 1 & 0 \\
\hline Diarrhea & 3 & 0 \\
\hline Constipation & 9 & 0 \\
\hline Anorexia & 13 & 3 \\
\hline Rash & 3 & 0 \\
\hline Fever & 5 & 0 \\
\hline Febrile neutropenia & 4 & 4 \\
\hline Heart failure & 0 & 0 \\
\hline Dyspnea & 6 & 1 \\
\hline Mucositis & 6 & 2 \\
\hline Malaise & 13 & 0 \\
\hline Neuropathy & 3 & 0 \\
\hline
\end{tabular}

Alb, Albumen; ALT, alanine aminotransferase; AST, aspartate aminotransferase; Cre, creatinine; Hb, hemoglobin; Plt, platelet count; T.Bil, total bilirubin, WBC, white blood cell.

Our intention was to reduce the frequency of adverse events, especially febrile neutropenia, which sometimes leads to treatment-related death, by modifying the treatment schedule to weekly administration from conventional administration on three consecutive days. However, $20 \%$ of the patients in this study developed febrile neutropenia and we were unable to show that weekly administration was safer than the conventional regimen. In conclusion, this study failed to demonstrate that weekly amrubicin has the same efficacy as conventional amrubicin therapy and no further development of this new regimen is warranted.

\section{Acknowledgements}

The Authors thank all of the patients and their families, as well as the investigators who participated in this study.

\section{References}

1 Yana T, Negoro S, Takada M, Yokota S, Takada Y, Sugiura T, Yamamoto H, Sawa T, Kawahara M, Katakami N, Ariyoshi Y, Fukuoka M and West Japan Thoracic Oncology Group: Phase II study of amrubicin in previously untreated patients with extensive-disease small cell lung cancer: West Japan Thoracic Oncology Group (WJTOG) study. Invest New Drugs 25: 253258, 2007.

2 Onoda S, Masuda N, Seto T, Eguchi K, Takiguchi Y, Isobe H, Okamoto H, Ogura T, Yokoyama A, Seki N, Asaka-Amano Y, Harada M, Tagawa A, Kunikane H, Yokoba M, Uematsu K, Kuriyama T, Kuroiwa Y, Watanabe K and Thoracic Oncology Research Group Study 0301: Phase II trial of amrubicin for treatment of refractory or relapsed small-cell lung cancer: Thoracic Oncology Research Group Study 0301. J Clin Oncol 24: 5448-5453, 2006.

3 Inoue A, Sugawara S, Yamazaki K, Maemondo M, Suzuki T, Gomi K, Takanashi S, Inoue C, Inage M, Yokouchi H, Watanabe H, Tsukamoto T, Saijo Y, Ishimoto O, Hommura F and Nukiwa $\mathrm{T}$ : Randomized phase II trial comparing amrubicin with topotecan in patients with previously treated small-cell lung cancer: North Japan Lung Cancer Study Group Trial 0402. J Clin Oncol 26: 5401-5406, 2008.

4 Ettinger DS, Jotte R, Lorigan P, Gupta V, Garbo L, Alemany C, Conkling P, Spigel DR, Dudek AZ, Shah C, Salgia R, McNally R, Renschler MF and Oliver JW: Phase II study of amrubicin as second-line therapy in patients with platinumrefractory small-cell lung cancer. J Clin Oncol 28: 2598-2603, 2010.

5 Jotte R, Conkling P, Reynolds C, Galsky MD, Klein L, Fitzgibbons JF, McNally R, Renschler MF and Oliver JW: Randomized phase II trial of single-agent amrubicin or topotecan as second-line treatment in patients with small-cell lung cancer sensitive to first-line platinum-based chemotherapy. J Clin Oncol 29: 287-293, 2011.

6 Norton L: Theoretical concepts and the emerging role of taxanes in adjuvant therapy. Oncologist 6(Suppl 3): 30-35, 2001.

7 Fennelly D, Aghajanian C, Shapiro F, O'Flaherty C, McKenzie M, O'Connor C, Tong W, Norton L and Spriggs D: Phase I and pharmacologic study of paclitaxel administered weekly in patients with relapsed ovarian cancer. J Clin Oncol 15: 187-192, 1997.

8 Belani CP, Ramalingam S, Perry MC, LaRocca RV, Rinaldi D, Gable PS and Tester WJ: Randomized, phase III study of weekly paclitaxel in combination with carboplatin versus standard every-3-weeks administration of carboplatin and paclitaxel for patients with previously untreated advanced non-small-cell lung cancer. J Clin Oncol 26: 468-473, 2008.

9 Kitagawa C, Saka H, Kajikawa S, Mori K, Oki M and Suzuki R: Phase I and pharmacologic study of weekly amrubicin in patients with refractory or relapsed lung cancer: Central Japan Lung Study Group (CJLSG) 0601 trial. Cancer Chemother Pharmacol 69: 1379-1385, 2012.

10 Eisenhauer EA, Therasse P, Bogaerts J, Schwartz LH, Sargent D, Ford R, Dancey J, Arbuck S, Gwyther S, Mooney M, Rubinstein L, Shankar L, Dodd L, Kaplan R, Lacombe D and Verweij J: New response evaluation criteria in solid tumours: revised RECIST guideline (version 1.1). Eur J Cancer 45: 22847, 2009. 
11 National Cancer Institute: Common Terminology Criteria for Adverse Events (CTCAE), Version 4.0. Available at: http:// evs.nci.nih.gov/ftp1/CTCAE/About.html. Accessed on 27 September 2018.

12 Inoue A, Sugawara S, Maemondo M, Mori Y, Oizumi S, Harada M, Taima K, Morikawa N, Ishida T, Kinoshita I, Watanabe H, Suzuki T, Nakagawa T, Saito R and Nukiwa T: Randomized phase II trial comparing amrubicin with re-challenge of platinum doublet in patients with sensitive-relapsed small-cell lung cancer: North Japan Lung Cancer Study Group trial 0702. Lung Cancer 89: 61-65, 2015.
13 Murakami H, Yamamoto N, Shibata T, Takeda K, Ichinose Y, Ohe Y, Yamamoto N, Takeda Y, Kudoh S, Atagi S, Satouchi M, Kiura K, Nogami N, Endo M, Watanabe H and Tamura T: A single-arm confirmatory study of amrubicin therapy in patients with refractory small-cell lung cancer: Japan Clinical Oncology Group Study (JCOG0901). Lung Cancer 84: 67-72, 2014.

Received August 2, 2018 Revised September 28, 2018 Accepted October 1, 2018 